Y. Shikata

Nagoya Math. J.

Vol. 156 (1999), 159-169

\title{
ON GEOMETRIC VARIATION THEORY
}

\author{
YOSHIHIRO SHIKATA
}

\begin{abstract}
We construct here a framework for a geometric variation of 1 dimensional geometric figures regarding them as sets of ordered points. In this framework, we can make full use of cut and paste technique to find a way to go down to the geometric smallest figure, including the topological change of the parameter space. Therefore we can discuss practical problems like switching of current flows and the minimal networks not only multiple closed geodesics.
\end{abstract}

\section{Introduction}

Practically, it is important to find a smallest geometric figure under given condition, where "smallest" means mostly the least length or the least area. The length or area of a geometric figure is measured usually through a parametrization of the figure. But for example, a geometric figure of a closed geodesic can be covered many times, showing the difficulty to figure out a geometric figure from parametrizations and the measurement of length or area. For example, Klingenberg introduced fascinating ideas to pick up simple closed geodesics in the space of path and count them in connection with equivariant variation theory.

But there remains another difficulty in parametrization of geometric figures, because the theory of minimization of non-parametric method develops keeping the topological type of the parameter space fixed.

Take for example soap films spanned on 2 rings of wire flames. An experiment shows the soap film spanning the flames changes its topological type from that of two disks to cylinder when the position of the rings are varied. If the topological type of the parameter space for the variation theory is fixed to be either the disk or the cylinder, the theory can not describe the change of the topological type. Therefore it can not attain the true minimal, when a change in topological type of the parameter space is necessary.

A similar example of the difficulty, which is of dimension one, is the current flow problem. Set 2 (electric) positive poles $\mathrm{P}(1), \mathrm{P}(2)$ and 2 negative poles $\mathrm{N}(1), \mathrm{N}(2)$ in 3 space. Then the current flows along the geodesic

Received September 29, 1998. 
from $\mathrm{P}(1)$ to $\mathrm{N}(1)$ or $\mathrm{N}(2)$ and $\mathrm{P}(2)$ to $\mathrm{N}(2)$ or $\mathrm{N}(1)$, respectively. The flow switches when the distance between the poles are varied as the soap film changes its shape.

Both in the soap film example and in the current flow example, we see that the cut and paste technique of the geometric figure, and therefore a topological change of the parameter space, is necessary to attain the true minimal. In these examples, the handle body describes the switching of the topological type of the parameter space and attains the true minimal. Since the handle body is deduced from a quadratic form with suitable negative part, we see that on the critical level of the handle body construction, there appears a geometric figure with singularity, which can be dually parametrized by each of the topological type. This suggests us necessity to construct a geometric variation theory on the geometric figures to admit branching or cut and paste technique.

In order to treat this problem, various attempts are done as geometric measure theory. Parametrization for a geometric figure and the general construction of the measure on them is started by Whitney [W] and followed by Almgren, Fedrer, which are sometimes too delicate to apply concrete problems as the minimal networks. Moreover there is not assured the continuity of the length or area on the variation space of this type but only the semi-continuity, making the convergence argument complicated.

In this exposition, we construct a framework for the global variation space admitting branching flows for geometric figures of one-dimension, where cut and paste of local minimals can take place as in the soap film or electric current example. For example, we can show that by the cut and paste technique the multiply covered geodesic goes down the simple one along the branching gradient "flow" in this framework. We combine the classical method of finding geodesics in Seifert-Threlfall with the idea in Cech cohomology for this purpose, which we review in the following section.

\section{Classical variation and ordered set}

We review here the method of classical variation in the large for the geodesics connecting given 2 points and interpret them in terms of our scheme.

Let $M$ be a compact connected Riemannian manifold and take $\delta=$ $\delta(M)$ so that any 2 points of distance less than $\delta$ can be connected by the unique shortest geodesic. 
Taking 2 different points $\mathrm{V}(1), \mathrm{V}(2)$ in $M$, we consider the global existence problem of a geodesic joining $\mathrm{V}(1), \mathrm{V}(2)$.

For a piecewise smooth path $c(t)$ of length less than $N \delta$ joining $\mathrm{V}(1)$, $\mathrm{V}(2)$, introduce the parameter proportional to the arc length. Then divide the parameter interval into $N$ congruent segments. We have an ordered set of points $t(1), t(2), \ldots, t(N)$ in the parameter space and the point set $\{c(t(i))\}$ in $M$.

The classical method proceeds as follows:

0) First there is introduced a topology in the space of the piecewise smooth path by

$$
\operatorname{dist}\left(c(t), c^{\prime}(t)\right)=\max \operatorname{dist}\left(c(t), c^{\prime}(t)\right)+\left|L(c)-L\left(c^{\prime}\right)\right|
$$

1) The points $c(t(i-1))$ and $c(t(i))$ are joined by the geodesic segments to yield a map $G^{\prime}$ of $c$ to a path $G^{\prime}(c)$ in the path space.

2) Then the middle points of the geodesic segments of $G^{\prime}(c)$ are joined by the geodesic segments to have $G^{\prime \prime}(c)$, leaving fixed the ends points.

In $[\mathrm{ST}]$, it is proved that

THEOREM 1. The composition map $G=G^{\prime \prime} \cdot G^{\prime}$ is continuous and decreases the length $L(c)$ of $c$ in general:

$$
L(c) \geq L(G(c))
$$

The equality holds if and only if $c$ is a geodesic.

THEOREM 2. The set of the paths $G \cdots G(c)$ contains a converging sequence to a geodesic.

We notice that the argument above holds if a path $c$ is replaced by a set $\Omega$ of points $\{c(i), i=1,2, \ldots, N\}$ with an order.

Definition 1. For an ordered set of $N$-points $\{c(i)\}$ in $M$, we define the length $L(\{c(i)\})$ as follows:

$$
L(\{c(i)\})=\sum \operatorname{dist}(c(i), c(i+1))
$$


Definition 2. For ordered sets of $N$-points $\{c(i)\}\left\{c^{\prime}(i)\right\}$, we define the distance between them as follows:

$$
\operatorname{dist}\left(\{c(i)\},\left\{c^{\prime}(i)\right\}\right)=\max \left(\operatorname{dist}\left(c(i), c^{\prime}(i)\right)\right)
$$

Proposition 1. The length function is continuous on the set of ordered points with respect to the topology induced by the distance function.

Proof. From the triangle inequality, it is obvious that

$$
\begin{aligned}
& \operatorname{dist}(c(i), c(i+1)) \\
\leq & \operatorname{dist}\left(c(i), c^{\prime}(i)\right)+\operatorname{dist}\left(c^{\prime}(i), c^{\prime}(i+1)\right)+\operatorname{dist}\left(c^{\prime}(i+1), c(i+1)\right) \\
\leq & \operatorname{dist}\left(c^{\prime}(i), c^{\prime}(i+1)\right)+2 \operatorname{dist}\left(\{c(i)\},\left\{c^{\prime}(i)\right\}\right) .
\end{aligned}
$$

Thus we have

$$
L(\{c(i)\}) \leq L\left(\left\{c^{\prime}(i)\right\}\right)+2 N \operatorname{dist}\left(\left\{c^{\prime}(i)\right\},\{c(i)\}\right) .
$$

In the similar way we have

$$
L\left(\left\{c^{\prime}(i)\right\}\right) \leq L(\{c(i)\})+2 N \operatorname{dist}\left(\{c(i)\},\left\{c^{\prime}(i)\right\}\right) .
$$

Hence we see that

$$
\left|L(\{c(i)\})-L\left(\left\{c^{\prime}(i)\right\}\right)\right| \leq 2 N \operatorname{dist}\left(\left\{c^{\prime}(i)\right\},\{c(i)\}\right) .
$$

Definition 3. An ordered set of points $\{c(i)\}$ is said to be $\delta$-ordered set or simply $\delta$-set, if the set satisfies that

$$
\operatorname{dist}(c(i), c(i+1))<\delta .
$$

We denote the totality of the $\delta$-ordered set by $\Omega(\delta)$.

The followings are proved in the similar way to the classical cases:

Proposition 2. For a $\delta$-ordered set $\{c(i)\}$, there corresponds a path $j(\{c(i)\})$, obtained by joining the points $c(i)$ and $c(i+1)$ by the geodesic segments. The correspondence $j$ is continuous with respect to the distance and the topology in the path space. The length $L(j\{c\})$ of the path agrees with $L(\{c\})$, the length defined for the ordered set. Conversely, for a piecewise smooth path $c(t)$ of length less than $N \delta$, there corresponds continuously a $\delta$-ordered set $\{c(i)\}=k(c(t))$ by $c(i)=c(t(i))$ as in the classical construction, using the parameter proportional to the arc length. 
Proposition 3. As a result of the composition with $j, k$, the mapping $G^{\prime}, G^{\prime \prime}, G$ in the classical construction are well defined as continuous mapping from $\delta$-ordered set $\{c(i)\}$ into the set of $\delta$-ordered set $\{c(i)\}$. We use the same notation for these maps provided there is no confusion.

TheOREm 3. The mapping $G$ decreases the length $L(\{c\})$ of $\delta$-ordered set $\{c(i)\}$ in general:

$$
L(\{c\}) \geq L(G(\{c\}))
$$

The equality holds if and only if $j(c)$ is a geodesic.

THEOREM 4. The set of the paths $G \cdots G(\{c\})$ contains a converging sequence to a geodesic.

\section{Geometric figure and parametrization}

We have seen that the parametrization of the path determines the order in the $\delta$-ordered set of points. Since we are interested in the geometric figure, which is independent of the parametrization, we introduce a mechanism to make the points set free from the order in this simplest case of the path.

Definition 4. For an ordered point set $P=\{P(i)\}$ in the manifold $M$, the set $|P|$ denotes its geometric figure, the order is suppressed.

Consider the set of all the permutation $\Pi$ on the set $\{2,3, \ldots, N-1\}$, then define the action of $\Pi$ on the set of the point set $P=\{p(i), i=$ $1,2, \ldots, N\}$ by

$$
\pi P=\{p(\pi(i))\} \quad \text { for any } \pi \in \Pi \text {. }
$$

For an ordered point set $P=\{p(i)\}$ derived from a parametrization, the set $\pi P$ corresponds to all the possibility of the parametrization for the given path with fixed boundary.

Definition 5. The permutation $\pi$, which gives the minimal of the length $L(\pi P)$ among $\pi \in \Pi$ is called the minimal permutation or simply $m$-permutation and the length is called $m$-length for the ordered set $P$.

Definition 6. For a geometric figure $|P|$ obtained from the ordered set $\{P(i)\}$, the length $L(|P|)$ is defined to be the $m$-length of $\{P\}$.

LEMMA 1. Suppose that the m-permutation $\pi$ is unique for the ordered point set $P$, then there exists a neighborhood $U$ of $P$ such that for any point $P^{\prime}$ in $U$, then m-permutation is also unique and given by $\pi$. 
Proof. Since $\pi$ is assumed to be unique, $\pi$ is effective at the same time and the length $L(\pi P)$ attains true minimal. Then Proposition 1 implies that for small $\varepsilon>0$, there exists a neighborhood $V$ of $\pi P$, where it holds that for $P^{\prime} \in U$ and $\pi^{\prime} \in \Pi\left(\pi^{\prime} \neq \pi\right)$

$$
L(\pi P)-\varepsilon<L\left(P^{\prime}\right)<L(\pi P)+\varepsilon<L\left(\pi^{\prime} P\right)-\varepsilon .
$$

Since $\Pi$ is finite, we can apply Proposition 1 also to $\pi^{\prime \prime} P$ for $\pi^{\prime \prime} \in \Pi$ to have

$$
L\left(\pi^{\prime \prime} \pi P\right)-\varepsilon<L\left(\pi^{\prime \prime} P^{\prime}\right)<L\left(\pi^{\prime \prime} \pi P\right)+\varepsilon<L\left(\pi^{\prime \prime} \pi^{\prime} P\right)-\varepsilon
$$

on a neighborhood $U$. Combining them we have

$$
L(\pi P)-\varepsilon<L\left(P^{\prime}\right)<L(\pi P)+\varepsilon<L\left(\pi^{\prime} P\right)-\varepsilon<L\left(\pi^{\prime \prime \prime} P^{\prime}\right)<L\left(\pi^{\prime} P\right)+\varepsilon
$$

for $\pi^{\prime \prime \prime}$ not equal to the identity. Thus we see that $P^{\prime}$ is minimal.

Remark. In general, we can not assume uniqueness of the $m$-permutation. In this case we replace it by a subset of the permutation, and deduce a similar conclusion.

COROllary 1. The lemma above implies that the set of $P$ where the uniqueness of m-permutation holds is open.

Definition 7. For geometric figures $|\{P(i)\}|,\left|\left\{P^{\prime}(i)\right\}\right|$ obtained from ordered sets $\{P(i)\},\left\{P^{\prime}(i)\right\}$, we define the distance between them as the minimal of the distance under the permutation:

$$
\operatorname{dist}\left(|\{P(i)\}|,\left|\left\{P^{\prime}(i)\right\}\right|\right)=\inf \left(\operatorname{dist}\left(\pi\{P(i)\}, \pi^{\prime}\left\{P^{\prime}(i)\right\}\right)\right)
$$

Remark. Generically it is not necessary to apply the permutation both variable $P, P^{\prime}$ to define the distance. In the application, we can fix the order in one of the variables.

Proposition 4. With respect to the topology induced by the distance, the length function on the set of geometric figures turns out to be a continuous function.

Proof. Without loss of generality, we can assume the ordered set $P$ gives the $m$-length. Taking then the neighborhood in Lemma 1 if the order is unique, the assertion follows directly. A similar argument finishes the general case. 
We introduce an abstract situation to consider the minimum of the length function on the space of the geometric figure.

Definition 8. A triple $(X, f, H)$ of a topological space $X$, a continuous function $f$ on it and a correspondence $H$ is said to be a variation triple, if it satisfies that

1) $f(H(x)) \leq f(x)$ for any $x$ in $X$

2) if $f(H(x))=f(x)$ then $H(x)=x$.

THEOREM 5. If the space $X$ is compact, the variation triple $(X, f, H)$ contains a fixed point for $H$.

Proof. Since $X$ is compact, for any point $x$ in $X$ the infinite sequence $\{H \cdots H(x)\}$ contains a subsequence converging to $x^{\prime}$. Though we do not know about the continuity of $H$, we have $f\left(H\left(x^{\prime}\right)\right)=f\left(x^{\prime}\right)$ from the continuity of $f$, thus condition 2 ) yields the conclusion.

We construct a variation problem on the set of geometric figures $\Omega / \Pi$ for the length function as follows:

For a geometric figure $|P|$ in $\Omega / \Pi$, we take its lift in $\Omega$, consisting of $\pi P$ for $\pi$ in $\Pi$. Choose a point $P^{\prime}$ in the lift, giving the $m$-length, then send $P^{\prime}$ by the map $G$ in $\Omega$ which decreases the length. Define the correspondence $H$ in $\Omega / \Pi$ as to send $P$ to the projection of $G\left(P^{\prime}\right)$ into $\Omega / \Pi$, which decreases the length on $\Omega / \Pi$.

If we assume the compactness in the underlying manifold $M$ then we see that $\Omega / \Pi$ is compact, thus together with Proposition 4 , we see that the triple $(\Omega / \Pi, L, H)$ forms a variation triple, and therefore Theorem 5 implies the correspondence $H$ has the fixed point.

Proposition 5. Let $M$ be a compact Riemannian manifold, then the triple of space $\Omega / \Pi$, the length function and the correspondence $H$ forms a variation triple on a compact space, thus $H$ has a fixed point in $\Omega / \Pi$.

Together with Theorem 1, we see that the set of fixed points of $H$ contains the true minimal geometric figure among that connected by a single path, because we took the space $\Omega$ big enough and considered there all the possible parametrization of a geometric figure. But we remark that in order to go down to the true global minimal only by $H$, we need further consideration in the choice of the starting set of paths. 
The difference between the global minimal in our framework and the ordinary geodesic can be seen in case of multiply covered geodesic $g(n t)$ of the simple closed geodesic $g(t), n>1$ as follows:

Since the gradient flow induced from the energy function $[K]$, which corresponds to the length, has all the geodesics as the critical points, the counting problem of the simple closed geodesics became very complicated. But as a result of the deal with the branching in the "flow", we can eliminate the multiple closed geodesics of even order from the critical point of the above variation triple, identifying that of odd order to the simple one on the other hand.

In fact, chop the simple geodesic $g(t)$ into $N$-points $\{g(t(i)), i=$ $1, \ldots, N\}$ and use these points to chop the multiple geodesic $g(n t)$. Take $n=2$ for example, then all the points are doubly counted, so that $g(t(i))=$ $g(t(i+N))$. Taking suitable points $P, Q$ among them, we can divide the geodesic $g(t)$ as $g^{\prime} \cdot P Q \cdot g^{\prime \prime}$ where $P Q$ implies the shortest geodesic joining them and the $g^{\prime}, g^{\prime \prime}$ is the part of $g$ before $P$ and after $Q$. Then the path from the origin to $P$ along $g^{\prime}$ and back along the inverse to $g^{\prime}$ then go to $Q$ along inverse to $g^{\prime \prime}$ and back along $g^{\prime \prime}$ is shorter than the double of the geodesic. Therefore it can be an image of the "flow" $H$, indicating that the multiple geodesic can not be the critical point in this variation triple.

The above construction technique of a shorter path in the image of $H$ generalized and will be named in case of a geometric figure with complex boundary as the mutation and discussed in the following section.

\section{Splitting of order system: strict splitting}

We do not go into the full detail of general non-sense of the construction of the abstract variation space, but illustrate it for typical cases to include the examples in the introduction.

Let $N$ denote the set of integers $1, \ldots, n$, which will be identified to the ordered points set consisting of $n$-points in $M$. The subset $\{1,2, n-1, n\}$ of 4 points is said to be the boundary $B$ of $N$.

Definition 9. A pair of ordered sets $U, V$ is said to be the strict splitting of $N$ bounding the boundary set, if they are imbedded in $N$ so that the images which again denoted by $U, V$ are disjoint and cover $N$ and if the point in the boundary set is covered by the set of first point or end point of $U, V$ :

$$
U \cap V=\Phi, \quad U \cup V=N
$$


\{first point and end point of $U$, first point and end point of $V\}=\{1,2$, $n-1, n\}$. We denote the set of the strict splitting by $\Sigma$.

We define length and the distance for the splitting as in Definitions 1, 2 as the sum of the length and the distance on each of the ordered set of the splitting using the permutation $\Pi$ in $N$ leaving set $B$ invariant. The length and the distance are independent of the order and the way to cut it into 2 parameter spaces, thus they correspond to the geometric figure bounding the set $B$ by 2 paths.

We denote the space of the set of $n$-points in $M$ containing $B$ by $\Omega(B)$. Since the space $\Omega(B)$ is covered further by the set $\Sigma(B)$ of the splitting, we see that the variation of geometric figures of this type and therefore the cut and paste technique is made possible in the space $\Sigma(B)$, especially the exchange of the boundary points.

LEMMA 2. Arrange the ordered sets $U, V$ of the strict splitting as $\{u(i)\},\{v(i)\}$, respectively. If there are pairs of points $u(i), u(i+1) ; v(i)$, $v(i+1)$ on each of the splitting such that

$$
\begin{aligned}
& \operatorname{dist}(u(i), v(i))+\operatorname{dist}(u(i+1), v(i+1)) \\
&<\operatorname{dist}(u(i), u(i+1))+\operatorname{dist}(v(i), v(i+1)),
\end{aligned}
$$

then the ordered sets $\{u(1), \ldots, u(i), v(i), v(i-1), \ldots, v(1)\} ;\left\{u\left(n^{\prime}\right)\right.$, $\left.u\left(n^{\prime}-1\right), \ldots, u(i+1), v(i+1), \ldots, v\left(n^{\prime \prime}\right)\right\}$ has the shorter length than that for the splitting $U, V$, thus can be a candidate of the "flow" $H$, where $n^{\prime}, n^{\prime \prime}$ stands for the end points of the ordered sets $U, V$, respectively.

Intuitively, we can say that a strict splitting and the "flow" $H$ describe how to cut the geometric figure of shape " $X$ " to obtain two paths joining four points $\{1,2, n-1, n\}$, in this sense it may be called the mutation. Lemma 2 corresponds a technique how to paste them together to yield a new parametrization and a geometric figure joining given four points.

In fact, take the example of the paths connecting the case of 2 (electric) positive poles $\mathrm{P}(1), \mathrm{P}(2)$ and 2 negative poles $\mathrm{N}(1), \mathrm{N}(2)$ in Introduction, the switching from the paths joining $\{\mathrm{P}(2), \mathrm{N}(1)\},\{\mathrm{P}(1), \mathrm{N}(2)\}$ change to the paths connecting $\{\mathrm{P}(1), \mathrm{N}(2)\},\{\mathrm{P}(2), \mathrm{N}(1)\}$ can be described in this setting.

In this case, the current may flow along a path from $\mathrm{P}(1)$ to $\mathrm{N}(1)$ and a path from $\mathrm{N}(2)$ and $\mathrm{P}(2)$, the chopping them into ordered sets of points 
yields a splitting. If the paths cross at a point, then there could be two types of the following splitting: One splitting is what corresponds to paths going through the crossing point from $\mathrm{P}(1)$ to $\mathrm{N}(1)$ and from $\mathrm{N}(2)$ to $\mathrm{P}(2)$. Another splitting is what corresponds to the path going from $\mathrm{P}(1)$ to the crossing then turning back to $\mathrm{N}(2)$ and the path from $\mathrm{N}(1)$ to the crossing then back to $\mathrm{P}(2)$.

As in this example, we see that a geometric figure can be parametrized in many ways and that the cut and paste technique is necessary to have the true minimal, which happens frequently in practical applications as in the examples in the introduction.

As far as to cut a geometric figure into 2 paths concerns, we see that the strict splitting gives the effective variation theory on geometric figures, in fact, the construction of $H$ for single ordered sets applies directly to the splitting. Therefore we have:

Proposition 6. Let $M$ be a compact Riemannian manifold, then the triple of space $\Sigma(B) / \Pi$, the length function and the correspondence $H$ forms a variation triple on a compact space, thus $H$ has a fixed point in $\Sigma(B) / \Pi$.

\section{Final remark for splitting in general}

In general, we have to cut a geometric figure into more than 2 pieces of paths to parametrize it. The situation corresponds to a covering $\{U(i), i=$ $1,2, \ldots, k\}$ of the set $N$, which may not necessarily be disjoint, similar to the construction of Cech cohomology.

We illustrate here only the typical case of covering $U, V$ such that

$$
U \cap V=W \neq \phi
$$

The splitting of this type corresponds to the geometric figure of shape " $H$ ", which plays the core role in the theory of network.

Definition 10. Let $W=\{w(1), \ldots, w(k)\}$ be an ordered subset of a set $U$, the order in $W$ is said to extend to that in $U$, if the element of $U$ is so arranged that

$$
U=\{u(1), \ldots, u(l), w(1), \ldots, w(k), u(l+1), \ldots, u(n)\}=\left\{U^{\prime}, W, U^{\prime \prime}\right\}
$$

Definition 11. A covering $U, V$ of $N$ by ordered sets is said to be the splitting of $N$, if both of the order in $U, V$ extends an order in $W=U \cap V$. 
Thus for a splitting $U, V$ of $N$, there are found mutually disjoint ordered sets $W, U^{\prime}, U^{\prime \prime}, V^{\prime}, V^{\prime \prime}$. We essentially consider the variation triple of pieces $W, U^{\prime}, U^{\prime \prime}, V^{\prime}, V^{\prime \prime}$ adding new boundary points $w(1), w(k)$ and apply the cut and paste technique.

The permutation to construct the "flow" $H$ is the partial permutation in each of them leaving the boundary invariant. We then define the length and the distance of the splitting as the sum of the length and the distance on these ordered sets using this permutation $\Pi^{\prime}$ of $N$.

We denote the space of the splitting by $\Sigma^{\prime}(B) / \Pi^{\prime}$, then as in the case of the strict splitting, we can construct a variation theory on geometric figures. Therefore we have:

Proposition 7. Let $M$ be a compact Riemannian manifold, then the triple of space $\Sigma^{\prime}(B) / \Pi^{\prime}$, the length function and the correspondence $H$ forms a variation triple on a compact space, thus $H$ has a fixed point in $\Sigma^{\prime}(B) / \Pi^{\prime}$.

Remark. The above variation triple does not give the true minimal in general, because there still is involved 2 variables not yet decided. In order to go down to the true global minimal, we need to consider the local variation process to vary these boundary points. Thus we see that a combination of the classical variation method $G$, global cut and paste method $H$ and the local analysis of the boundary completes the global variation for the network theory on a manifold, to which we return in the next exposition.

\section{REFERENCES}

[K] W. Klingenberg, Lectures on closed geodesics, Springer-Verlag, 1978.

[ST] H. Seifert and W. Threlfall, Variationsrechnung im Grossen, Leipzig: Teubner, 1938.

[W] H. Whitney, Geometric integration theory, Princeton University Press, 1956.

Department of Mathematics

Meijo University

Shiogamaguchi, Tenpaku-ku

Nagoya 468-8502

Japan

shikat@meijo-u.ac.jp 